Research Paper

\title{
Anatomical Analysis on the Lateral Bone Window of the Sella Turcica: A Study on 530 Adult Dry Skull Base Specimens
}

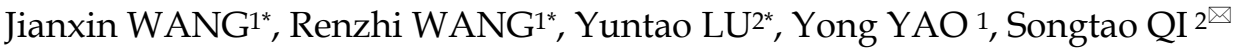 \\ 1. Department of Neurosurgery, Peking Union Medical College Hospital, Chinese Academy of Medical Sciences, Beijing 100730, China \\ 2. Department of Neurosurgery, Nanfang Hospital, Southern Medical College, Guangzhou 510515, China \\ * These authors (Jianxin WANG, Renzhi WANG and Yuntao LU) contributed equally to this work and are the co-first authors of this article.
}

$\triangle$ Corresponding author: Songtao QI: Department of Neurosurgery, NanFang Hospital, Southern Medical University, NO.1838 Guangzhou avenue of Baiyun District, Guangzhou 510515 China. Tel.: 0086-20-61641801; fax: 0086-20-61641806. Email:qisongtao_smu@163.com

(c) Ivyspring International Publisher. This is an open-access article distributed under the terms of the Creative Commons License (http://creativecommons.org/ licenses/by-nc-nd/3.0/). Reproduction is permitted for personal, noncommercial use, provided that the article is in whole, unmodified, and properly cited.

Received: 2013.07.10; Accepted: 2013.12.12; Published: 2014.01.03

\begin{abstract}
Objective: To investigate the morphometric characteristics of the lateral bone window (LBW) of the sella turica.

Methods: A descriptive anatomical study of LBW was performed in 530 cases of dry skull base specimens with relatively complete sella turcica. Detailed morphometric characteristics such as dimensions and calssification of the LBW was studied. All data analysis was performed using SPSS I7.0 statistical software.

Results: LBW is located in the lateral bony structures of sella turcica. The mean area sizes of the LBW were $75.99 \pm 25.81 \mathrm{~mm}^{2}$ (left) and $76.00 \pm 25.53 \mathrm{~mm}^{2}$ (right). There was no significant difference and there was a good positive correlation between bilateral areas of the LBWs. The area size of the LBWs is graded as follows: Grade A $\left(<60 \mathrm{~mm}^{2}\right), B\left(60 \sim 90 \mathrm{~mm}^{2}\right)$ and C $\left(>90 \mathrm{~mm}^{2}\right)$. LBW morphology was typed as follows: Type I, II, III, IV. And Tpye III includes Type IIla and IIIb; Type IV includes Type IVa, IVb, IVc, IVd.

Conclusions: The lateral bone window of sella turcica is an important structure located between pituiary fossa and parasellar region. The morphological measurements and variations of LBW in this study will provide preliminary data for further anatomical study of sella turcica. Moreover, knowing detailed anatomy of this region is essential for neurosurgeons who make surgery on cranial base or for teaching about the sella turcica in the neuroanatomy lab.
\end{abstract}

Key words: Anatomy; Lateral bone window; Sella turcica; Clinoid process; Sella turcica bridge; Clinical significance

\section{Introduction}

Located at the center of the skull base and characterized by its complex anatomical structure, its variable morphology and its close relationship with different anatomical structures including cranial nerves, pituitarygland, and cavernous part of the internal carotid artery, sella turcica has ever been regarded as a forbidden zone in neurosurgery. Through developments in microusurgical techniques and imaging techniques such as MRI and CT, neurosurgeons become more aware and better understanding of the pathologic anatomy of this region. This awareness and understanding provoked neurosurgeons to make anatomical research on this region and then related reports are increasing [1-10]. However, so far, there has been few numbers researches on the part of the lateral bone window (LBW) of the sella turcica ( a window, located in the lateral bony structure of the sella turcica, formed by the line between the anterior and posterior clinoid processes, the inner edge of the carotid sulcus, the posterior border of the optic strut, 
and the lateral border of dorsum sellae, and etc.). As this structure has close relationship with the pituitary fossa and parasellar region, LBW is worth studying.

We performed a descriptive anatomical study of 530 adult dry skull base specimens, results of the morphological measurements and variations have been commented to provide preliminary data for further anatomical study of the sella turcica, to aid to understand the potential influence of anatomical factors of sella turcica on the growth pattern of intrasellar tumours and further to help neurosurgeons make optimized operative plans for sellar lesion.

\section{Materials and methods}

Five hundred thirty dry skull base specimens with relatively complete sella turcica of Chinese adults (300 males and 230 females, mean age 67 years, range 20-98 years) were collected for the study. The following characteristics of the LBW (Table 1) were measured or assessed:

i) measurement of the area sizes of both LBWs, and analysis of the correlation between LBW area and depth of the sella turcica (STD)

ii) observation and measurement of the distance between the anterior and posterior clinoid processes (APD)

iii) observation and measurement of the maximum transverse diameter (MTD) of LBW

iv) observation and measurement of the relative ratio between APD and MTD

v) observation and measurement of the maximum longitudinal diameter (MLD) of LBW and the relative ratio between $M L D$ and the height of the middle clinoid process (MCP)

vi) observation and measurement of the occurrence and morphological characteristics of the sella turcica bridge (STB) and MCP

vii) preliminary grading and classification of LBW, based on relevant data and morphology and

viii) measurement of the lateral bone height ( $\mathrm{LBH}$, the vertical distance between the highest point on the bottom bone edge of LBW and the sellar floor).

Of all the characteristics above, it is most difficult to measure the area size of LBW due to its irregularity. We used plasticine, vernier calipers, a camera, and ImageJ software (National Institutes of Health, USA) to measure LBW area (Figure 1). We filled the sella with plasticine to demarcate the boundaries of LBW, and then removed the plasticine to observe contours of the LBW imprinted there. The upper boundary was defined by drawing a line from the anterior clinoid process to the posterior clinoid process, or by the lower edge of STB, when present. We affixed a multi-angle scale marker to the plasticine and took a photo, making certain that the camera lens was parallel with the plane of LBW, in order to reduce measurement error. Next, we used the "irregular shapes" function in our ImageJ software to draw a line along the contour of LBW. We then selected and measured the shape to get an initial value of the LBW area. The actual value of the LBW area was calculated proportionally using the scale marker. All data analysis was performed using SPSS 17.0 statistical software (Statistical Product and Service Solutions, USA). The means of measurement data were compared with independent samples $t$-test or paired $t$-test. Data correlations were analyzed using Pearson correlation. The criterion for statistical significance for all tests was $p<$ 0.05 .

We confirmed that these tissue samples were donated to science and there was a detailed donation registration file for each specimen. And all measurement procedures were performed in accordance with guidelines issued by the Committee on Research in Human of Peking Union Medical College Hospital and approved by the Institutional Ethics Committee (Peking Union Medical College, Beijing, China).

Table I. English abbreviations of indicators observed in the present study

\begin{tabular}{|l|l|}
\hline Abbreviation & Full name \\
\hline LBW & Lateral bone window of sella turcica \\
\hline APD & $\begin{array}{l}\text { Distance between anterior clinoid and poste- } \\
\text { rior clinoid processes }\end{array}$ \\
\hline MTD & Maximum traverse diameter \\
\hline MLD & Maximum longitudinal diameter \\
\hline STB & Sella turcica bridge \\
\hline MCP & Middle clinoid process \\
\hline LBH & $\begin{array}{l}\text { Height of lateral bone of pituitary fossa } \\
\text { Depth of sella turcica }\end{array}$ \\
\hline CCFH & Carotico-clinoid foramen of Henle \\
\hline
\end{tabular}

\section{Results}

\section{Concept, location, and composition of LBW}

The left and right LBWs are like windows located in the lateral bony structure of the sella turcica (Figure 2). The upper boundary is the line between the anterior and posterior clinoid processes, while the lower boundary is the inner edge of the carotid sulcus. The anterior boundary of LBW is the posterior border of the optic strut, and the posterior boundary is the lateral border of dorsum sellae. Sometimes, a STB or MCP exists inside LBW, and when an STB is present, the upper boundary of LBW is the lower edge of STB. When $\mathrm{MCP}$ and anterior clinoid process are completely ossified, the carotico-clinoid foramen of Henle (CCFH) is forming [11], and the anterior boundary of LBW is the anterior border of CCFH. 


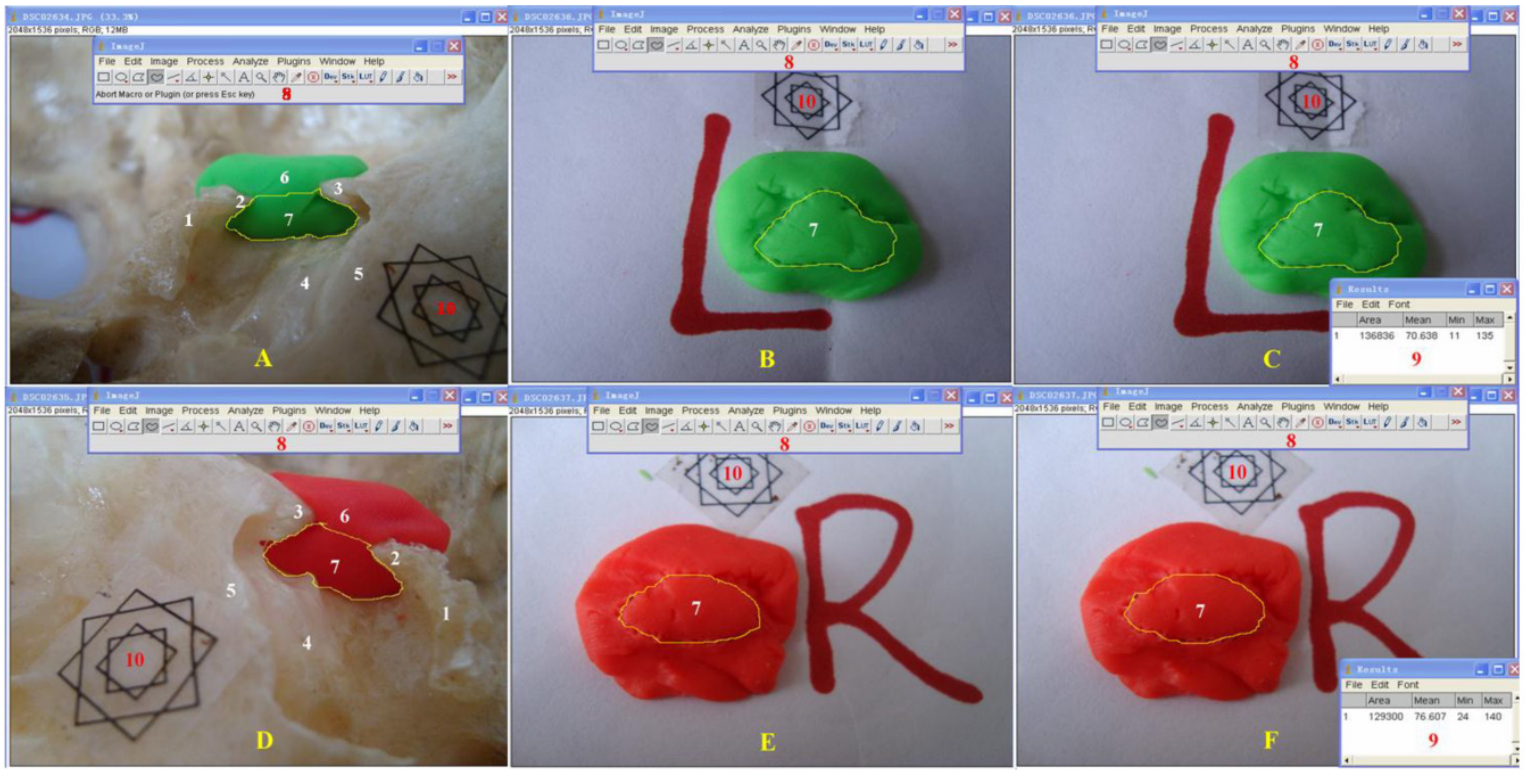

Figure I. The procedure used to calculate the area of the lateral bone window as shown by the ImageJ software interface. I. dorsum sellae; 2 . posterior clinoid process; 3 . anterior clinoid process; 4 . tuberculum sellae; 5 . planum sphenoidale; 6 . plasticine; 7 . scope of the lateral bone window (LBW); software toolbar; 9. calculated result column; I0. Multi-angle scale Marker; L: left side; R: right side

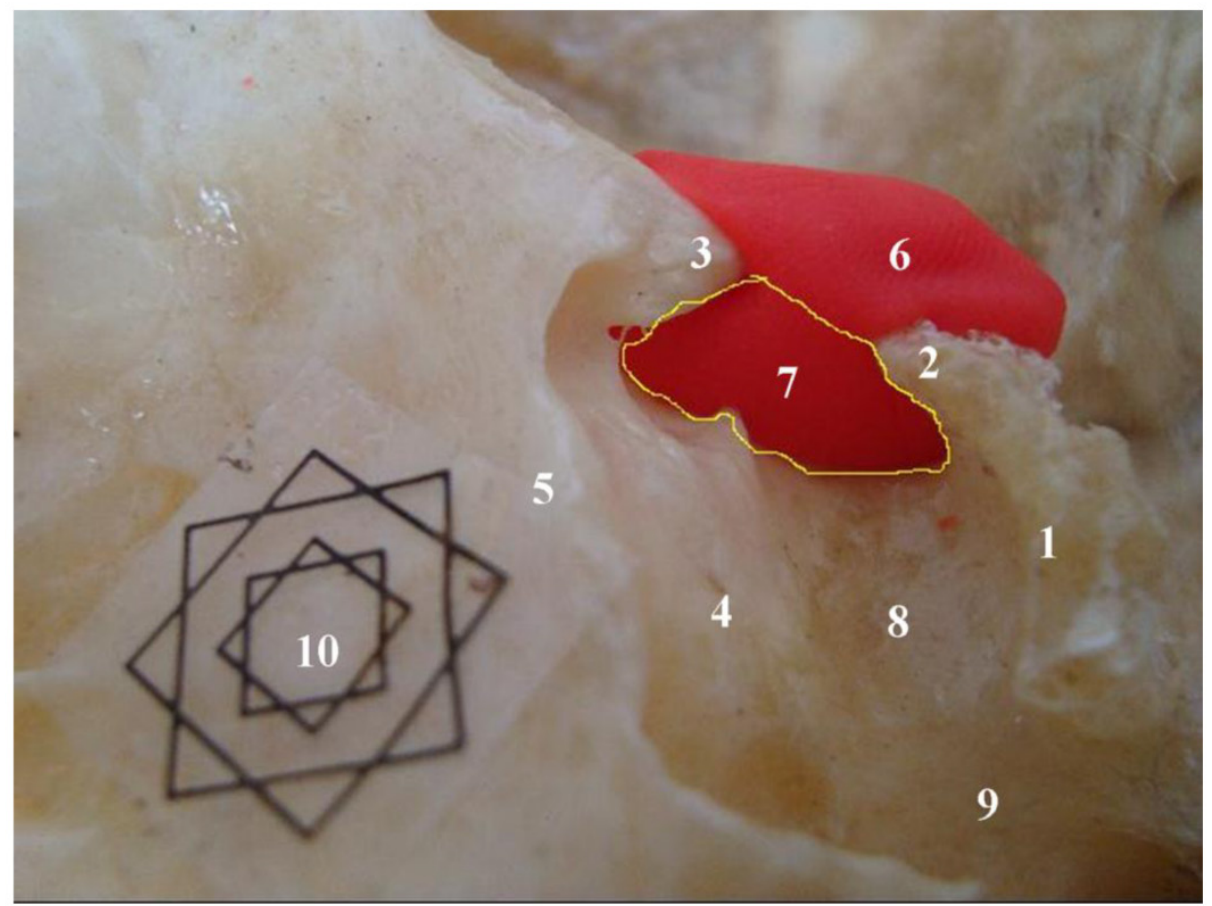

Figure 2. Specimen showing the lateral bone window (LBW) (the scope within the yellow line). I. dorsum sellae; 2. posterior clinoid process; 3. anterior clinoid process; 4. tuberculum sellae; 5 . planum sphenoidale; 6. plasticine; 7. lateral bone window (LBW); 8. sellar floor; 9. carotid sulcus (CAS); 10. Multi-angle scale Marker (external: $1 \mathrm{~cm}$; internal: $0.5 \mathrm{~cm}$ )

\section{Classification of the area of LBW}

The mean measured area size of the left LBW was $75.99 \pm 25.81 \mathrm{~mm}^{2}$, the right was $76.00 \pm 25.53$ $\mathrm{mm}^{2}$, and the mean STD was $11.00 \pm 1.82 \mathrm{~mm}$. There was a positive correlation between the areas of the left and right LBWs (Pearson correlation analysis, $\mathrm{r}=$ $0.638, p=0.000$ ) and the area of LBWs and STD (left $\mathrm{r}=$ $0.214, p=0.000$; right $r=0.180, p=0.000)$. We as- signed grades based on the measured area size of the LBWs as follows: Grade A-LBW area less than $60 \mathrm{~mm}^{2}$ (20\% of the subjects); Grade B-LBW area between 60 $\mathrm{mm}^{2}$ and $90 \mathrm{~mm}^{2}(60 \%)$; Grade C-LBW area greater than $90 \mathrm{~mm}^{2}(20 \%)$.

\section{Morphology of LBW}

We considered three main factors when assessing LBW morphology: APD, MCP, and the for- 
mation of the STB. The mean left APD measured was $7.28 \pm 3.13 \mathrm{~mm}$, and the mean right APD was $7.29 \pm$ $3.29 \mathrm{~mm}$. The mean left MTD was $19.83 \pm 1.87 \mathrm{~mm}$, and the mean right MTD was $20.45 \pm 1.84 \mathrm{~mm}$. The ratio (R) of APD to MTD can be used to control the relative openness of LBW. The complete LBW type, with $\mathrm{R}<1: 4$, was found in $20 \%$ of the subjects. The semi-open type, with $R$ between $1: 4$ and 1:2, was found in $60 \%$ and the open type, with $R>1: 2$, was found in $20 \%$. The shape of MCP can be divided into three types: spinous, protruding and cylindrical types. The cylindrical type is approximately the same height as MLD. The incidence of MCP was 36.79\% ( $16.04 \%$ on the left, and $20.75 \%$ on the right side). Most MCPs were located within the anterior third of the lower LBW. Most MCPs were less than a third of the height of the MLD height. The second most common were MCPs of the same height as MLD, and least common were MCPs of a height near half of MLD (Table 2). The STB is defined as a "bony connection between the anterior and posterior processes" [12]. To meet our research needs, we modified this definition in order to include bony connections among any clinoid process. The STB should therefore consist of several types: pure anterior clinoid to posterior clinoid process type, anterior clinoid to middle clinoid to posterior clinoid process type, pure anterior clinoid to middle clinoid process type, and pure middle clinoid to posterior process clinoid type (Table 3). In our study, the incidence of STB was $9.62 \%$, with occurrence on the right slightly more common than on the left. The pure anterior clinoid to posterior clinoid process type and the anterior clinoid to middle clinoid to posterior clinoid process type belong to the complete STB group, with an incidence of $5.18 \%$ (1.98\% on the left and $3.20 \%$ on the right). There was no pure middle clinoid to posterior clinoid process type, so we can divide STBs into two categories: full-STB (5.18\%) and semi-STB (7.73\%). Full-STB includes the pure anterior clinoid to posterior clinoid process type, and the anterior clinoid to middle clinoid to posterior clinoid process type. Semi-STB includes only the pure anterior clinoid to middle clinoid process type. Both the anterior clinoid to middle clinoid to posterior clinoid process type STB and the pure anterior clinoid to middle clinoid process type STB form the CCFH [11], which are equal of the complete type and/or contact type CCFH in the typing described by Keyes [13].

Based on the above results, we summarized LBW morphology as follows (Figure 3): MTD.

Type I (open type): APD is longer than half of

Type II (semi-open type): APD is longer than one-quarter of MTD, but shorter than half of MTD

Type III (intact type): including relatively intact type (IIIa) and absolutely intact type (IIIb). The former occurs when the APD is less than one-quarter of MTD, and the latter occurs when the APD is equal to zero (when STB exists)

Type IV (latch-like type): MCP height is equal to MLD, and is like the latch of a door in relation to LBW. We can further subclassify this type according to MCP shape and size combined with the CCFH in Keyes classification $[7,13]$

Type IVa: equivalent to the complete CCFH of Keyes typing (including a complete bony connection between the anterior and posterior clinoid processes).

Type IVb: equivalent to the contact $\mathrm{CCFH}$ of Keyes typing (including an incomplete bony connection between the anterior and posterior clinoid processes).

Type IVc: equivalent to the incomplete CCFH of Keyes typing (including a space between the anterior and posterior clinoid process)

Type IVd: intact LBW with complete CCFH.

\section{Measurement of LBH}

The lateral bone of the pituitary fossa lies at the lower edge of the LBW, like the threshold of a door. No statistical significant difference was detected between the lateral bone height (LBH) on the left (mean, $1.23 \pm 0.79 \mathrm{~mm}$ ) and right (mean, $1.22 \pm 0.82 \mathrm{~mm}$ ) sides $(p=0.166)$ (Figure 4$)$.

Table 2. Occurrence of middle clinoid processes (MCPs) $(n=530)$.

\begin{tabular}{|c|c|c|c|c|c|c|c|c|c|}
\hline \multirow[t]{2}{*}{ Sides } & \multicolumn{3}{|c|}{ Location* } & \multicolumn{3}{|c|}{ Height** $^{* *}$} & \multicolumn{3}{|c|}{ Shape ${ }^{* * *}$} \\
\hline & A1/3 & M1/3 & P1/3 & Same & Half & Lower 1/3 & Spiny & Projective & Cylindrical \\
\hline Left & 54 & 26 & 0 & 25 & 4 & 52 & 39 & 16 & 22 \\
\hline Right & 106 & 9 & 0 & 30 & 1 & 83 & 30 & 54 & 33 \\
\hline Total & 160 & 35 & 0 & 55 & 5 & 135 & 69 & 70 & 55 \\
\hline Ratio (\%) & 30.19 & 6.60 & 0 & 10.38 & 0.94 & 25.47 & 13.02 & 13.21 & 10.38 \\
\hline
\end{tabular}

Note: A1/3: anterior 1/3 segment; M1/3: middle 1/3 segment; P1/3: posterior 1/3 segment.

* Referring to the location of MCP relative to the bottom margin of LBW.

** Referring to the height of MCP relative to the MLD of LBW.

***The shapes include spiny, projective and cylindrical MCPs, and the cylindrical is the highest 
Table 3. Occurrence of sella turcica bridges (STBs) $(n=530)$.

\begin{tabular}{|l|l|l|l|l|}
\hline Type & Left & Right & Bilateral & Total \\
\hline $\mathrm{AP}^{*}$ & $10(1.89 \%)$ & $4(0.75 \%)$ & $5(0.09 \%)$ & $19(3.58 \%)$ \\
\hline $\mathrm{AM}^{* *}$ & $5(0.09 \%)$ & $13(2.45 \%)$ & $5(0.09 \%)$ & $23(4.34 \%)$ \\
\hline $\mathrm{AM}^{* * *}$ & $21(3.96 \%)$ & $20(3.77 \%)$ & $10(1.89 \%)$ & $51(9.62 \%)$ \\
\hline $\mathrm{MP}^{* * * *}$ & $0(0.00 \%)$ & $0(0.00 \%)$ & $0(0.00 \%)$ & $0(0.00 \%)$ \\
\hline Total & $36(6.79 \%)$ & $37(6.98 \%)$ & $20(3.77 \%)$ & \\
\hline
\end{tabular}

Note: *AP: The pure anterior clinoid to posterior clinoid processes type

${ }^{* *}$ AMP: The anterior clinoid to middle clinoid to posterior clinoid processes type

***AM: The pure anterior clinoid to middle clinoid processes clinoid type

**** MP: The pure middle clinoid to posterior clinoid process type

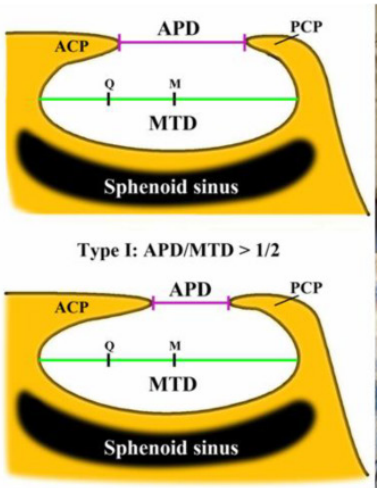

Type II: $1 / 4<$ APD/MTD $<1 / 2$

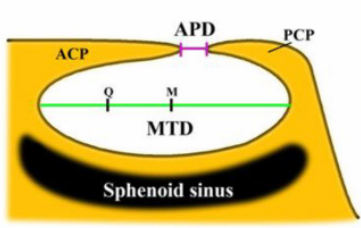

Type IIIa: APD/MTD $<1 / 4$

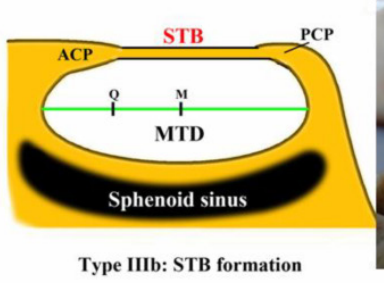

Type IIIb: STB formation
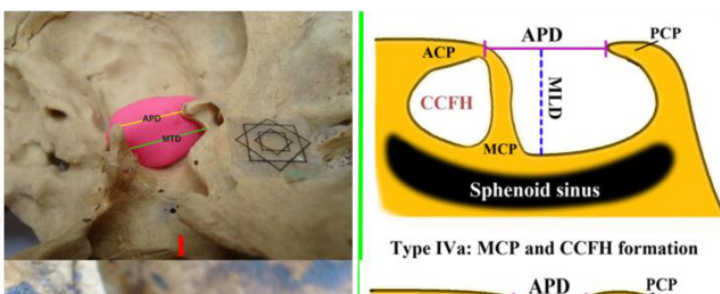

Type IVa: MCP and CCFH formation

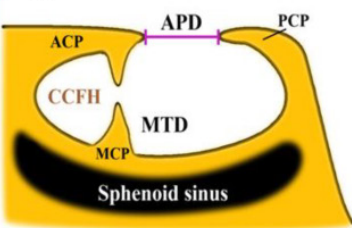

Type IVb: Imperfect MCP and CCFH

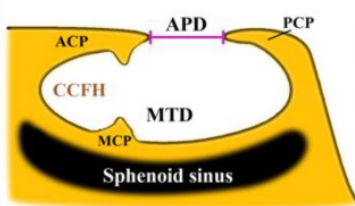

Type IVc: Partial MCP and CCFH

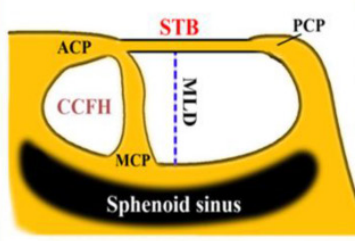

Type IVd: STB, MCP and CCFH formation

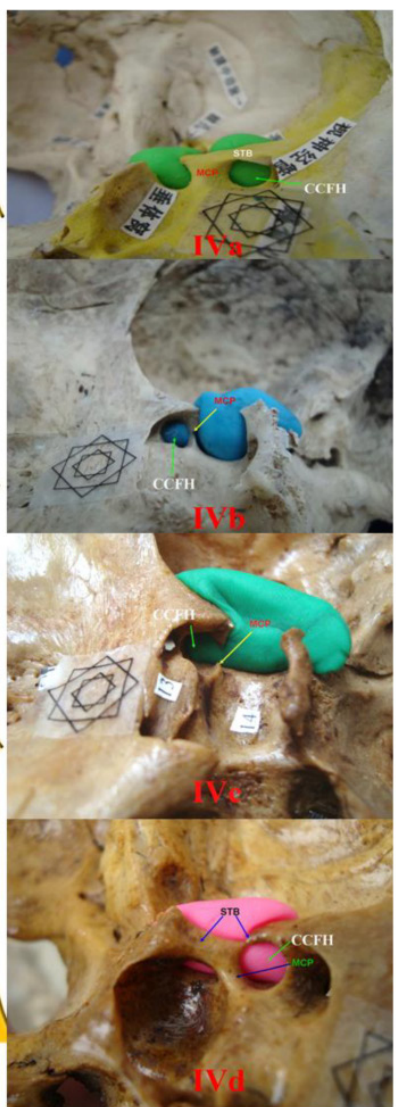

Figure 3. Lateral bone window (LBW) shape classifications in diagrammatic (left) and photographic (right) formats. According to the relative size of the APD, occurrence of STB, and occurrence of MCP or types of CCFH, LBW can be typed into four types as follows: I- open type: IIhalf-open type; III- complete type; IV- latch-like type; A: anterior clinoid process; P: posterior clinoid process; APD: distance between anterior clinoid and posterior processes; MTD: maximum transverse diameter of LBW; Q: quarter split point of MTD; M: mid-point of MTD; STB: sella turcica bridge; MLD: maximum longitudinal diameter of LBW; MCP; middle clinoid process; CCFH: carotico-clinoid foramen of Henle; LBW: lateral bone window of sella turcica
Figure 4. Diagram of the lateral bone height (LBH) (reflecting the height of the threshold of the bottom margin of the LBW). ACP; anterior clinoid process; LBH: lateral bone height of pituitary fossa; LBW: lateral bone window of sella turcica; MLD: maximum longitudinal diameter of LBW; CAS: carotid sulcus

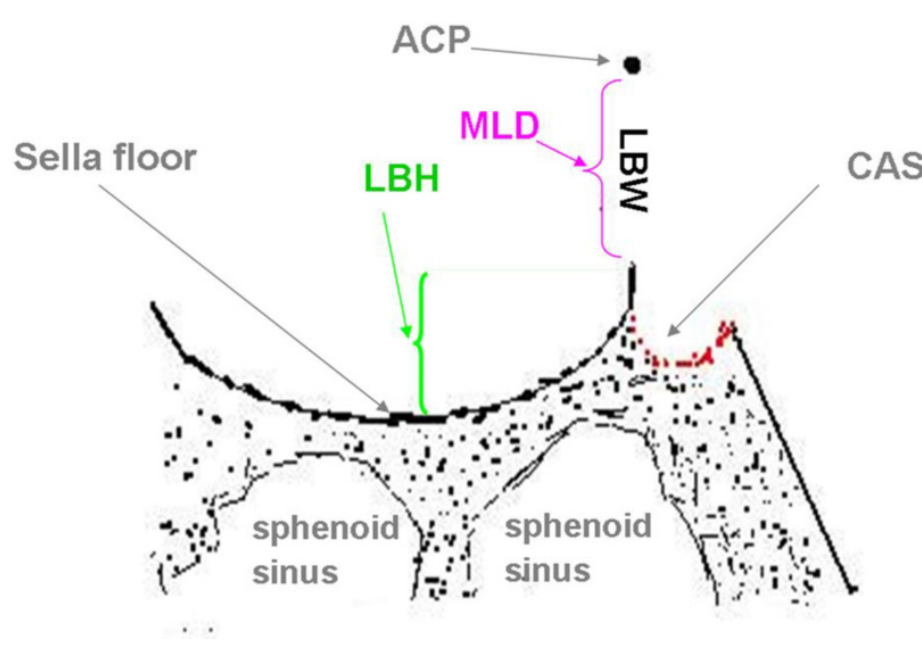




\section{Discussion}

The majority of previous studies of the lateral wall of the sella turcica have focused on the anterior and posterior clinoid processes and on the CCF [9,13-14]. No study has estimated the morphologic anatomy of LBW. However, as LBW is an important structure located between pituitary fossa and parasellar region, its anatomical and morphological characteristics are worth studying. In this study, specific values of LBW size were successfully measured and correlations were found between the left and right LBWs and between LBW and STD. We speculated that the area size of LBW might have something to do with the resistance to objects passing through LBW from pituitary fossa to parasellar region due to its acting as an important channel. When the area size of LBW becomes large, ie. the channel from pituitary fossa to parasellar region would become large and smooth, and it should be beneficial to the intrasellar lesions' expansion into parasellar regions. When LBW size is particularly small, the growth direction of pituitary adenomas might be expected to be up or down, without parasellar extension. The weak positive correlation between LBW and STD might indicate that the deeper the STD is and the larger the LBW area should be. Pherhaps when the sella turcica is deeper and the intrasellar lesions are more likely to grow through LBW, because the area size of LBW also became larger accordingly. In addition, it seems to be relatively easier to remove the lesions inside cavernous sinus through LBW with large area than through LBW with very small area via the medial wall of cavernous sinus during transsphenoidal surgery, because the former could provide wider operation space than the latter.

We described LBW morphology based on three factors: the length of APD, MCP and STB formation.

As regards to APD, few reports categorize the sella turcica or its related structures with reference to APD. A Chinese report [15] classified sella turcica in three types based on APD. In open type, the size of APD was greater than $5 \mathrm{~mm}$ in $39 \%$ of subjects, in closed type, APD size less than $2 \mathrm{~mm}$ in $57 \%$ and in semi-open type, APD size between $2 \mathrm{~mm}$ and $5 \mathrm{~mm}$ in $4 \%$. Based on our findings, we estimate that the previous study has some limitations. Firstly, it classifies the sella turcica with reference only to APD, without considering the morphology of the lateral bony wall. Another limitation is that the clinical significance of this classification was not highlighted and LBW size was not considered. For example, when APD size is more than $5 \mathrm{~mm}$, and LBW is similarly large, it is hard to believe that the sella turica belongs to the open type. A third limitation is that it requires specific numerical values for APD in order to determine sella turcica type, making this method of classification inefficient. Our classification system considers both APD and LBW, not requiring specific numerical values, but only the relative ratio of APD/MTD. This relative ratio can be obtained after gross observation, making this classification more practical. The APD is just like a "skylight" located in the upper LBW, and its size is closely related to the size of the contact area between intrasellar lesions and the cavernous sinus, and as well to the degree of difficulty of parasellar extension through the LBW. When the APD is relatively large, LBW is relatively open and the contact surface between the lesions and parasellar structures is relatively large. A large APD facilitates the pituitary adenomas' growth into the cavernous sinus.

As regards to $\mathrm{MCP}$, it is an inconsistent small bony protrusion of the sphenoid bone, located 1-2 mm below the tuberculum sellae $[10,16]$. It appears a wide range of incidence from 41.4 to $75 \%$ [15, 17]. A lower incidence of MCP $(36.79 \%)$ was found in our study comparing with the incidence reported in the literature. The MCP incidence on the right side was higher $(20.75 \%)$ than that on the left $(16.04 \%)$. In this study, the morphology of MCP can be described as three types (Table 3): spiny, projective, and the cylindrical types. As to its location, the MCP was most often located in the anterior third of the bottom edge of LBW and less commonly found in the middle third, while no case was found in the posterior third. This finding comes in agreement with the definition of MCP as this entity is located lateral inferior to tuberculum sella. As to the height of $\mathrm{MCP}$, we have not measured the exact height of $\mathrm{MCP}$, but the relative height of the MCP to MLD. The most common type is MCP of less than lower third of the MLD, the second most common is MCP of the same height as MLD (most of these MCPs are cylindrical type), and the least common is MCP of a height near half of MLD. The MCP is within LBW, just like a latch inside a door. It is easy to understand that the existence of this "latch" might increase the difficulty of parasellar adenoma extension. And this difficulty in extension is increased with increasing height of $\mathrm{MCP}$. Especially for cylindrical MCP, it constitutes $10.38 \%$ of all MCPs and has the same height as MLDs in the present stduy. The cylindrical MCPs can provide more resistance for parasellar extension than the spiny and projective MCP types. This might indicate that MCP height should be an important factor affecting the parasellar extension of pituitary adenomas.

As regards to STB, the incidence varies in different studies. Camp [8] found STBs in 4.5\% (5/110) of the cases. Chang et al. [18] and Scheithauer et al. [19] based on 461 and 1040 radiographs reported an incidence of $4.6 \%$ and $7 \%$, respectively. Keyes [13] re- 
ported an incidence of $8.86 \%$, after the examination of the bridge between the anterior and posterior processes. The STB incidence rate in our study was higher than that in previous reports. We speculate that there are several possible reasons for this variability incidence rates. Some studies used head radiographs as research material. In radiography, the overlapping structures or the fuzzy two-dimensional images provide a false positive or false negative representation and as result a false estimation of STB occurrence. In addition, each research is based in different study-group (the selected subjects are based on different inclusion criteria) and in some studies the gender of the population was not considered. The size of the sample also varied considerably among the research groups. Finally, the definition of STB is different among the studies. In our study, we included STBs of the pure anterior clinoid to middle clinoid process type, which may be the main reason that our STB incidence rate was higher than that of previous reports. STB acts as a bar lying transversely on the upper openning of the LBW, serving as another barrier preventing parasellar extension of pituitary adenomas. Because the incidence of STB in our study was almost $10 \%$, STB should be considered when studying factors that influence the growth pattern of pituitary adenomas. In addition, the STB type between the anterior and middle clinoid processes has the potential to harm the carotid artery during anterior clinoidectomy [20].

Based on these findings, we have classified the morphology of LBW as type I-open type, type II-semi-open type, type III-intact type (subdivided into relatively intact IIIa and absolutely intact IIIb) and type IV-latch-like type (subdivided into IVa, IVb, IVc and IVd subtypes) (Figure 3). This classification has the following characteristics: i) As the first classification system for LBWs, it can provide a basis for future research on sella tuicica; ii) Several factors influencing the morphology of LBW were taken into considersion in our classification system for LBWs. The factors include ACP, MCP and STB; iii) It is easy to use and identify the various types of LBW after gross observation of the anatomical specimens or using three-dimensional image reconstruction specimens. Evaluating LBW with the above system has a great clinical significance.

The anatomic characteristics of LBW can influence the intrasellar tumors' expansion to the adjacent parasellar region. The resistance to tumor extension gradually increases from types I to type III, especially in the subtype IIIb with a complete STB. The existence of STB or a narrow APD relative to MTD strengthens the boundary of LBW. The size of APD also might determine the area of contact between the pituitary and parasellar tissues. The MCP acts as a door latch within LBW, especially for type IV, it can greatly increase the difficulty of parasellar extension of pituitary adenomas through LBW. Moreover, when performing an operation in sellar region, it will also be important for surgeons to define whether MCP and STB exist preoperatively in order to decrease the occurrence of complications.

Besides the structures obove, we also found that there is a bony structure of variable height on both sides of the pituitary fossa. This lateral bone lies at the bottom edge of LBW. The LBH is equivalent to a gate "threshold" within LBW (Figure 4). In theory, the LBH is the first barrier encountered during parasellar extension of intrasellar lesions, especially for prolactin and growth hormone adenomas, because these cells are mainly located in the lateral portions of the anterior lobe of the pituitary gland [11,17]. When the depth of the pituitary fossa is deep and then LBH looks very high, the threshold effect of LBH as to LBW will be more obvious.

Currently, the reasons why some tumours grow upward and why some infiltrate cavernous sinus are still unclear. Cadaveric studies can only help us to estimate it. Studying of ligamentous structures around the pituitary gland have been found to be attractive because thin and defective ligamentous structures seem to play roles in the pathogenesis of pituitary adenoma extensions. It has been confirmed that weak diaphragm sella [21] or defective medial side of the cavernous sinus [22] may play a role in pituitary adenoma extention. Moreover, there were extracellular matrix studies of the medial wall of cavernous sinus [23], MRI studies [24] and studies evaluating the biological behaviour clinically and using labelling index of the pituitary adenomas [25-29] and its influence on cavernous sinus invasion.

According to our results in the present study, combined with the literatures concerned, we think that osseous variations of the sella turcica should be taken into consideration in addition to the some labelling index, the medial wall of cavernous sinus, and so on, when assessing the affecting factors on the parasellar growth of pituitary adenoma.

One limitation of this study lies in that the data of LBW were got from dry skull specimens (in vitro), not from the patients (in vivo). Extrapolation of data obtained from dry bone studies into clinical practice should be performed after meticulous consideration because of inherent differences between data of LBW in vrtro and in vivo. LBW in vivo is surrounded by connective tissue, blood vessels and neural structures. In our further study, we will combine closely the result of this study with clinical issues. 


\section{Abbreviations}

LBW: Lateral bone window of sella turcica; APD: Distance between anterior clinoid process and posterior clinoid process; MTD: Maximum traverse diameter; MLD: Maximum longitudinal diameter; STB: Sella turcica bridge; MCP: Middle clinoid process; LBH: Height of lateral bone of pituitary fossa; STD: Depth of sella turcica; CCFH: Carotico-clinoid foramen of Henle

\section{Conclusion}

The lateral bone window of sella turcica is an important structure located between pituiary fossa and parasellar region. The morphological measurements and variations of LBW in this study will provide preliminary data for further anatomical study of sella turcica. Moreover, knowing detailed anatomy of this region is essential for neurosurgeons who make surgery on cranial base or for teaching about the sella turcica in the neuroanatomy lab.

\section{Acknowledgments}

The authors thank Professors Jin Shi, Zuyuan Ren and Changbao Su for their valuable comments and kind support throughout this study.

\section{Competing Interests}

The authors have declared that no competing interest exists.

\section{References}

1. Renn WH, Rhoton AL Jr. Microsurgical anatomy of the sellar region. J Neurgsurg 1975; 43: 288-298.

2. Bergland RM, Ray BS, Torack RM. Anatomical variations in the pituitary gland and adjacent structures in 225 human autopsy case. J Nurosrug 1968; 28 : 93-99.

3. Gulsen S, Dinc AH, Unal M, Cantürk N, Altinors N. Characterization of the anatomic location of the pituitary stalk and its relationship to the dorsum sellae, tuberculum sellae and chiasmatic cistern. J Korean Neurosurg Soc 2010; 47:169-173.

4. Rhoton AL Jr. The sellar region. Neurosurgery 2002; 51: S335-S374

5. Lee HY, Chung IH, Choi BY, Lee KS. Anterior clinoid process and optic strut in Koreans. Yonsei Med J 1997; 38: 151-154.

6. Destrieux C, Kakou MK, Velut S, Lefrancq T, Jan M. Microanatomy of the hypophyseal fossa boundaries. J Neurosurg 1998; 88: 743-752.

7. Ozdoğmuş O, Saka E, Tulay C, Gürdal E, Uzün l, Cavdar S. The anatomy of the carotico-clinoid foramen and its relation with the internal carotid artery. Surg Radiol Anat 2003; 25: 241-246.

8. Camp J D. The normal and pathologic anatomy of the sella turcica as revealed at necropsy. Radiology 1923; 1: 65-73.

9. Kapur E, Mehić A. Anatomical variations and morphometric study of the optic strut and the anterior clinoid process. Bosn J Basic Med Sci 2012; 2: 88-93.

10. Narolewski R. Significance of anatomic variants of bony surroundings of the internal carotid artery and their significance for lateral surgical approaches to the cavernous sinus. Ann Acad Med Stetin 2003; 49: 205-229.

11. FitzPatrick M, Tartaglino LM, Hollander MD, Zimmerman RA, Flanders AE. Imaging of sellar and parasellar pathology. Radiol Clin North Am 1999; 37: 101-121.

12. Becktor JP, Einersen S, Kjaer I. A sella turcica bridge in subjects with severe craniofacial deviations. Eur J Orthod 2000; 22: 69-74.

13. Keyes JEL. Observations of four thousand optic foramen in human skulls of known origin. Arch Ophthalmol 1935; 13: 538-568.

14. Yasuda A, Campero A, Martins C, Rhoton AL Jr, Ribas GC. The medial wall of the cavernous sinus: microsurgical anatomy. Neurosurgery 2004. 55: 179-190.

15. Lu SC, Yang ZJ, Liao JC. Sphenoid sinus and pituitary adenoma. Shanghai, China: Shanghai medical university press; 2002: 33-34 (In Chinese)
16. Koshy JM, Bindhu S. Bilateral carotico-clinoid foramen and inter-clinoid bars. Recent Research in Science and Technology 2012; 4(7): 1-2.

17. Elster AD. Modern imaging of the pituitary. Radiology 1993; 187:1-14.

18. Chang CY, Luo CB, Teng MM, Guo WY, Chen SS, Lirng JF, Chang FC. Computed tomography and magnetic resonance imaging characteristics of giant pituitary adenomas. J Formos Med Assoc 2000; 99: 833-838.

19. Scheithauer BW, Kovacs KT, Laws ER Jr, Randall RV. Pathology of invasive pituitary tumors with special reference to functional classification. J Neurosurg 1986; 65: 733-744.

20. Isolan GR, de Aguiar PH, Laws ER, Strapasson AC, Piltcher O. The implications of microsurgical anatomy for surgical approaches to the sellar region. Pituitary 2009; 12: 360-367

21. Kursat E, Yilmazlar S, Aker S, Sksoy K, Oygucu H. Comparison of lateral and superior walls of the pituitary fossa with clinical emphasis on pituitary adenoma extension: cadaveric-anatomic study. Neurosurg Rev 2008; 31: 91-98.

22. Yilmazlar S, Kocaeli H, Eyigor O, Hakyemez B, Korfali E. Clinical importance of the basal cavernous sinuses and cavernous carotid arteries relative to the pituitary gland and macroadenomas: quantitative analysis of the complete anatomy. Surg Neurol 2008; 70: 165-174.

23. Knappe UJ, Fink T, Fisseler-Eckhoff A, Schoenmayr R. Expression of extracellular matrix-proteins in perisellar connective tissue and dura mater. Acta Neurochir (Wien) 2010; 152: 345-353.

24. Knosp E, Steiner E, Kitz K, Matula C. Pituitary adenomas with invasion of the cavernous sinus space: a magnetic resonance imaging classification compared with surgical findings. Neurosurgery 1993; 33: 610-618.

25. de Aguiar PH, Aires R, Laws ER, lsolan GR, Logullo A, Patil C, Katznelson L. Labeling index in pituitary adenomas evaluated by means of MIB-1: is there a prognostic role? A critical review. Neurol Res 2010; 32: 1060-1071.

26. Thapar K, Kovacs K, Scheithauer BW, et al. Proliferative activity and invasiveness among pituitary adenomas and carcinomas: Analysis using the MIB-1 antibody. Neurosurgery 1996; 38: 99-107.

27. Beaulieu E, Kachra Z, Mousseau N, Delbecchi L, Hardy J, Bélive au R. Matrix metalloproteinases and their inhibitors in human pituitary tumors. Neurosurgery 1999; 45: 1432-1441.

28. Kawamoto H, Uozumi T, Kawamoto K, Arita K, Yano T, Hirohata T. Type IV collagenase activity and cavernous sinus invasion in human pituitary adenomas. Acta Neurochir (Wien) 1996; 138: 390-395.

29. Knosp E, Kitz K, Steiner E, Matula C. Pituitary adenomas with parasellar invasion. Acta Neurochir Suppl (Wien) 1991; 53: 65-71. 